\title{
Zeitschriftenschau/Journal reviews
}

N.B.: Mit dem vorliegenden Heft beginnt die ZAC eine Zeitschriftenschau. Dabei ist eine vollständige Ubersicht über alle einschlägigen Erscheinungen auf dem Gebiet des antiken Christentums in seiner ganzen Breite weder beabsichtigt noch in Zeiten der fortgeschrittenen datentechnischen Möglichkeiten bibliographischer Hilfsmittel überhaupt notwendig. Eine ausführliche Auseinandersetzung mit den referierten Thesen der Autorinnen und Autoren soll in der Regel an dieser Stelle nicht stattfinden. Die Herausgeber möchten auf diese Weise bestimmte wichtige oder entlegen publizierte Beiträge (in der Regel Aufsätze, in Ausnabmefällen auch bedeutsame Rezensionen) einer größeren Leserschaft zur Kenntnis bringen und bitten daher um Zusendung von entsprechenden Sonderdrucken.

Hanns Christof Brennecke und Christoph Markschies

N.B.: With this volume on hand the ZAC starts a series of short journal reviews. A complete view of all relevant publications in the field of Ancient Christianity in full is neither intended nor necessary in view of today's electronic bibliographical information systems. In general a detailed discussion of the authors' theses is not intended. Thus the editors are planning to present certain important or not commonly known contributions (mainly papers, in exceptional cases also important reviews) to a broader readership. To this end we ask you to forward respective offprints to us.

\section{Hanns Christof Brennecke and Christoph Markschies}

A.N. Adkin, Jerome on Tertullian: Epist. LVIII 10,1, Athenaeum 87, 1999, 383-394. Untersucht wird die Äußerung: Tertullianus creber est in sententiis, sed difficilis in loquendo; es zeigt sich, daß das Thema ,Hieronymus und Tertullian' von C. Mohrmann keineswegs abschließend behandelt wurde (C. Mohrmann, Saint Jérôme et Saint Augustin sur Tertullien, VigChr 5, 1951, 111f.)

C.M.

T.D. Barnes, Rez. K. Sallmann (Hg.), Die Literatur des Umbruchs. Von der römischen zur christlichen Literatur 117-284 n.Chr., HLL IV, München 1997, in: Phoenix 52, 1998, 386-389.

Der Rezensent legt ebenso scharfsinnig wie scharfzüngig den Finger auf konzeptionelle und inhaltliche Probleme des Bandes, die bereits beim Titel beginnen. Ausgenommen werden davon allerdings die Beiträge von Tränkle, Heck, Gülzow, Doignon und Wlosok, denen (im Unterschied zu den Beiträgen über die Juristen) solide Qualität bescheinigt wird.

C.M.

N. Belayche, Dimenticare ... Gerusalemme. Les paganismes à Aelia Capitolina du $11^{\bullet}$ au IV' siècle de notre ère, REJ 158, 1999, 287-348.

Die Autorin versucht zu zeigen, daß zwischen der jüdischen Stadt aus der zweiten Tempelperiode und der neuen christlichen Stadt des vierten bis sechsten Jahrhunderts das Stratum einer heidnischen Stadt liegt, nqui présente à peu près toutes les charactéristiques d'une ville gréco-romaine d'Orient comme une autre" (S. 287). Dabei werden vor allem einschlägige Beiträge israelischer Archäologen zitiert und ausgewertet; es zeigt sich, daß auch in dieser Stadt versucht wurde, wie in einem Miniaturmodel! das stadtrömische religionsgeographische Vorbild zu imitieren (S. 300). Die komplexe Diskussion über die Frage, ob Juden im späteren zweiten und dritten Jahrhundert die 
Stadt faktisch wieder betreten durften und dort sogar lebten, wird in dem Aufsatz ebenfalls kurz aufgegriffen (S. 293f.): „Cette présence juive restait néanmoins très sporadique et Aelia Capitolina n'est plus une ville juive“ (S. 294).

C.M.

J. Dochhorn/A. Klostergaard Petersen, Narratio Ioseph: A Coptic Joseph-Apocryphon, JSJ 30, 1999, 431-463.

Die Autoren zeigen, daß das von J. Zandee publizierte Textstück aus dem Besitz von Carl Schmidt (Iosephus contra Apionem. An Apocryphal Story of Joseph in Coptic, VigChr 15, 1961, 193-213) nicht aus monastischen Kreisen Ägyptens stammt, sondern argumentieren "not only for a Jewish origin but for a Jewish content of the text as well" (S. 432). Sie weisen die Schrift der Gattung nrewritten Bible“ (G. Vermes) zu und stellen Parallelen in der antiken jüdischen exegetischen Literatur zusammen (S. 452-462): "There is no decisive reason to interpret Narr Ios in its present form as a Christian text ${ }^{\text {" }}$ (S. 463). Die Frage, ob es eine griechische Vorlage der sahidischen Narr los gab, ist dagegen nicht eindeutig zu entscheiden (S. 449).

C.M.

H. Donner/E. Sieg, Observations and Investigations in the Upper Valley of the Hermitage Near Ad-Dayr, Petra, ADAJ 42, 1998, 279-292.

Der Arrikel überprüft einige von G. Dalman Anfang des Jahrhunderts beschriebene Anlagen (ders., Petra und seine Felsheiligtümer. Palästinische Forschungen zur Archäologie und Topographie Bd. I, Leipzig 1908, 255-262; ders., Neue Petra-Forschungen und der Heilige Fels von Jerusalem. Palästinische Forschungen zur Archäologie und Topographie Bd. II, Leipzig 1912, 30f.). Dabei wird vorgeschlagen, Dalman nr. 441 mit seinem stibadium als Privatheiligtum oder als Aufbewahrungsort eines geheiligten Objektes zu deuten (S. 281f.); in der Höhle Dalman nr. 442 lebten ausweislich der Kreuze seit dem vierten Jahrhundert Mönche (S. 283f.). Vgl. nun auch Donners Bemerkungen in zwei weiteren Aufsätzen: Die Klause, die der Schlucht den Namen gab, in: U. Hübner u.a. (Hgg.), Nach Petra und ins Königreich der Nabatäer. Notizen von Reisegefährten. Für Manfred Lindner zum 80. Geburtstag, BBB 118, Bodenstein 1998, 110-119 sowie ders., Eine christliche Klause unterhalb des Plateaus von ed-Dër, Petra, am Kopf der sogenannten Klausenschlucht (BronNow/DomaszEwSKI Nr. 460 = DalmaN Nr. 424), ZDPV 115, 1999, 49-50.

C.M.

J. Elfassi, Le sermon 150 de saint Augustin, REAug 45, 1999, 21-50.

Der Herausgeber ediert die nun auch im Mainzer Fund (Stadtbibliothek 19, fol. $77^{\circ}$ 79v) belegte Predigt nach den diversen Zeugen kritisch (S. 39-49), bietet ein Stemma (S. 26) und orientiert über die historische Situation: Augustin predigte über Apg 17,18 vermutlich zwischen dem 15. und 21. Mai 397 (S. 31-38; nach Kunzelmann, Perler und Maier wesentlich später: S. 31 Anm. 45/46).

C.M.

A. Elli, Cristianesimo e Paganesimo in Egitto (IV-VI secolo), ScC 127, 1999, 616-648.

Auch wenn der Autor längst nicht alle wichtigen Titel kennt und verwendet, die in den letzten Jahren zu diesem spannenden Forschungsfeld verfaßt worden sind, vermag er durch seinen ganz aus den Quellen gearbeiteten Beitrag interessante Schlaglichter auf den Tempel von Philae (S. 624-629) und das alte Thema "La distruzione dei Templi pagani $^{4}$ (S. 629-635) zu werfen.

C.M. 
M.P. Foley, Cicero, Augustine, and the Philosophical Roots of the Cassiciacum Dialogues, REAug 45, 1999, 51-77.

Der Verfasser führt es auf eine verbreitete Geringschätzung der philosophischen Kompetenzen Ciceros in der Moderne (S. 52) zurück, daß die exakte Beziehung der Frühdialoge Augustins auf das Werk des römischen Autors bislang nicht zureichend erfaßt wurde: Augustins Contra Academicos stelle eine Antwort auf Ciceros Academica dar, De ordine auf Ciceros De finibus und die Tusculanae disputationes. Schließlich müsse De ordine als eine Antwort auf die Trilogie De natura deorum, De divinatione und De fato begriffen werden. Deswegen werden die Dialoge Augustins was a Christian response to classical Latin philosophy " verstanden, weniger als "a naive neo-Platonism or a defective embrace of the Christian faith" (S. 76).

C.M.

P. Grossmann, Besucher und Überfälle in der vorjustinischen Laura am Mosesberg: Zur Glaubwürdigkeit der diesbezüglichen Quellentexte im Hinblick auf die mitgeteilten topographischen Gegebenheiten, ByZ 92, 1999, $455-465$.

Die schwierige Frage nach der Glaubwürdigkeit der sogenannten relatio des Ammonius Monachus (CPG III, 6088; G. nennt eine auch im Supplement übersehene Edition: S. 457, Anm. 9, die im Katharinenkloster käuflich zu erwerben ist) und der narrationes des Nilus (CPG III, 6044; die BiTeu-Ausgabe von F. Conca im Supplementum), die allen vertraut ist, die sich einmal ausführlicher mit dem antiken palästinischen Mönchtum beschäftigt haben, wird von Grossmann erneut aufgeworfen. Dabei stellt er fest: „Die allgemeinen Rahmenbedingungen, die in den relationes des Ammonios mitgeteilt werden, sprechen (...) nicht gegen die Echtheit dieser Schrif" (S. 459). Er zeigt dies auch für die dramatischen Ereignisse, die Nilus erzählt (S. 464f.). - Ubrigens wird am Titelblatt der traditionsreichen byzantinischen Zeitschrift deutlich, daß der Verkauf des Verlages Teubner an den Verlag K.G. Saur (München/Leipzig) offenbar leider auch das traditionelle Signet ${ }_{\text {BGT }}{ }^{\text {}}$ zum Verschwinden gebracht hat, mit dem an Benedictus Gotthelf Teubner (1784-1856) erinnert wurde. Seit 1824 zierte es die altsprachlichen Texte des Verlages, der 1811 mit drei Handpressen begonnen hatte (F. Schulze, B.G. Teubner 1811-1911. Geschichte der Firma, Leipzig 1911), und überlebte mehrere Eigentümerwechsel.

Ch. Joest, Die pachomianische Geheimschrift im Spiegel der Hieronymus-Übersetzung. Mit dem deutschen Text von Brief $11 \mathrm{~b}$ des Pachomianischen Schriftencorpus und dem Versuch einer Úbertragung, Muséon 112, 1999, 21-46.

Der bereits durch eine ganze Reihe von Studien zum pachomianischen Mönchtum hervorgetretene Autor widmet sich dem eigenartigen Phänomen der Geheimschrift, indem er zunächst die überlieferten Geheimbuchstaben in allen Versionen der Briefüberlieferung dokumentiert und die Abweichungen zu erklären versucht (S. 25-35). Dabei kann der allein vollständigen lateinischen Uberlieferung kein gutes Zeugnis ausgestellt werden: „Das Ergebnis ist also [für Hieronymus, C.M.] wesentlich magerer: nur noch sieben Buchstaben geben einigermaßen treu das wieder, was in der Vorlage stand" (S. 35). Bei seiner anschließenden Deutung des Briefes 11b (S. 40f. 41-46) löst Joest die Buchstaben dann so auf, daß viele Schriftzitate entstehen (S. 41), und stellt eine "Liste der Deutungsvorschläge" zusammen (S. 38-40). 
H. Leppin, Constantius II. und das Heidentum, Athenaeum 87, 1999, 457-480.

Der Autor versucht in Fortsetzung der durch Barnes, Brennecke, Girardet und Klein vorgenommenen Neubewertung des Herrschers („revisionistisches Constantius-Bild": S. 458) dessen Verhältnis zum Heidentum neu zu beleuchten. Leppin zeigt, daß Constantius seit 350 christliche Symbole in seiner Selbstdarstellung nur mit Zurückhaltung verwendete (S. 461), und läßt seine Beamten sich in einer Sprache äußern, die Christen und Heiden gemeinsam ist (ebd.). "Kirchbauten wurden wohl nicht zwangsläufig als ausschließlich an Christen gerichtete Bauwerke aufgefaßt" (S. 462). Das Edikt Cod. Theod. XVI 10,4 vom 1. 12. 354 habe u.a. nur deswegen geringe Wirkung gezeigt, weil längst pagane Kreise das Opfer abgelehnt hätten (S. 471) und der Kaiser eine bestehende Entwicklung fortsetzte. - Den einen Herausgeber der Zeitschrift wollen wir nur ungern als ${ }_{n} \mathrm{H}$.J. ${ }^{*}$ vorgestellt sehen (S. 457).

C.M.

W. Portmann, Die politische Krise zwischen den Kaisern Constantius II. und Constans, Historia 48, 1999, 301-329.

Der Autor zeigt (u.a. in kritischer Wendung gegen Schwartz: S. 302 Anm. 10), daß der kirchenpolitische Streit das Verhältnis zwischen den Brüdern tief beeinflußte, und interpretiert dazu die 59. Rede des Libanius (S. 302-304) sowie die Münzserie FEL TEMP REPARATIO (S. 307-319), die vor dem Hintergrund der Versöhnung interpretiert wird, die in der zweiten Hälfte des Jahres 346 eintrat. Den Ausgangspunkt bildet die zutreffende Beobachtung, daB der jüngere Constans nach der Beseitigung Constantins II. etwa zwei Drittel des Reichsgebietes regierte (S. 302). „Constans' Funktionalisierung des kirchlichen Dissenses für seine eigenen Machtansprüche war bestimmend für die kaiserliche Politik in den Jahren 342-346. Sie scheint auch Auswirkungen auf die Politik der Sassaniden gehabt zu haben" (S. 329). Für die folgenden Jahre bis 350 spricht Portmann von einer unter "dem gemeinsamen Druck beider Kaiser" erzwungenen kirchlichen "Friedhofsruhe" (ebd.).

C.M.

J.-M. Prieur, La croix vivante dans la littérature Chrétienne du II siècle, RHPR 79, 1999, $435-444$.

Prieur stellt die Texte nochmals zusammen (S. 435-441), um dann zu zeigen, daß bei einigen Autoren des zweiten Jahrhunderts Leiden und Tod Christi nicht bedacht und systematisch reflektiert werden (S. 443). Das Motiv des personifizierten Kreuzes, wie es z.B. im Petrusevangelium auftritt, befindet sich also durchaus in einer Linie mit solchen Tendenzen, weil hier das Kreuz spiritualisiert wird und als Symbol verstanden wird (S. 443).

C.M.

C. Römer, Basilius, Epistula XXII 3 und das Glaubensbekenntnis des Gregor Thaumaturgos in einem Papyrus aus Antinoe, ZPE 123, 1998, 101-104.

Frau Römer interpretiert einen Papyrus, der in der Ausstellung über die Ausgrabungen von Antinoe 1998 in Florenz zu sehen war (PSI inv. 1213; aufgefunden 1937/1938). Sie zeigt, daß es sich um einen Beleg des Bekennenisses handelt, das von Gregor von Nyssa Gregor Thaumaturgus zugeschrieben wurde (CPG I, 1764), und vermutet, daß dieser verbreitete Text von einem Mönch zum Privatgebrauch abgeschrieben wurde. Das würde gut zu den diversen Passagen der Verbreitung und außerordentlichen Wertschätzung dieses Textes passen, die die Autorin zuverlässig gesammelt und in den Anmerkungen aufgeführt hat.

C.M. 
M.G. Schmidt, Ambrosii Carmen De Obitu Probi. Ein Gedicht des Mailänder Bischofs in epigraphischer Uberlieferung, Hermes 127, 1999, 99-116.

Der Autor versucht nachzuweisen, daß das anonym überlieferte Grabgedicht für Petronius Probus ICVR II, 4219b (= ILCV I, 63b), das vor dem Abbruch seines in Rom befindlichen Mausoleums unter Nikolaus $V$. noch abgeschrieben wurde, nalle für Ambrosius' literarisches Schaffen und theologisches Denken typischen Elemente" (S. 108) enthält. Die Zuweisung des Gedichtes an den Mailänder Bischof, in dessen Leben Probus eine wichtige Rolle spielte, ist durch die hier vorgelegten Argumente mindestens wahrscheinlich gemacht worden.

C.M.

P. Schüngel, Ein gnostisches Credo - in Stein gehauen!, RQ 93, 1998, 145-154.

Schüngel interpretiert zum wiederholten Male einen Text (in diesem Falle die in Rom aufbewahrte Inschrift ICVR VI, 297a) in Auseinandersetzung mit C. Scholten (Gibt es Quellen zur Sozialgeschichte der Valentinianer Roms?, ZNW 79, 1988, 244-261) und P. Lampe (An Early Christian Inscription in the Musei Capitolini, in: D. Hellholm/H. Moxnes/T. Karlsen Seim [Hgg.], Mighty Minorities? FS J. Jervell, Oslo 1995, 79-92) als Werk des römischen Theologen Valentin: „Es ist also sehr wohl denkbar, ja sogar ziemlich wahrscheinlich, daß er den Vierzeiler verfaßte; dieser wäre dann in die vierziger oder fünfziger Jahre des 2. Jahrhunderts zu datieren “ (S. 154).

C.M.

H. Tränkle, Textkritische Bemerkungen zu Augustins Confessiones, Hermes 127, 1999 , 209-236.

Zu diesem Aufsatz kann man nur dankbar hervorheben, was sein Verfasser einleitend bemerkt: „Die hier vorgelegten Bemerkungen sind hervorgegangen aus mehrfacher Beschäftigung mit dem ganzen Werk oder einzelnen Teilen davon im Laufe von etwa zwei Jahrzehnten“ (S. 208). Die Vorschläge unterstützen die Tendenz Skutellas (BiTeu, Stuttgart ${ }^{2} 1969$ ), sich von der Textgestaltung in der Edition Knölls (CSEL 33, Wien 1896) noch stärker abzusetzen. Zu der philologischen Dimension von O'Donnells verdienstvollem Kommentar fällt Tränkle ein kritisches Urteil und belegt es sogleich: $S$. 209 Anm. 7. Der Aufsatz kann auch als knappe Einführung in die stemmatischen Probleme der Schrift Augustins (S. 210f.) und in die Probleme der bisherigen Kollationen des handschriftlichen Materials gelesen werden.

C.M.

K.-H. Uthemann, Kaiser Justinian als Kirchenpolitiker und Theologe, Aug. 39, 1999 , 5-83.

In Form eines kleinen Kompendiums informiert der Autor über Person (S. 5f.), Kirchenpolitik und Theologie (S. 6-83). Das Henotikon stellt keine kirchenpolitische Wende dar (S. 10); die theopaschitische Formel wird als neine authentische Interpretation von Chalkedon" gedeutet und es werden Korrekturen an der Justinian-Deutung von Schwartz und Grillmeier vorgetragen (S. 16-24). Dabei sind Quellen und Literatur am Ende der jeweiligen Abschnitte übersichtlich zusammengestellt und die Darstellung klar pointiert. Man hätte diesen Beitrag gern in einem der großen gegenwärtig erscheinenden Lexika gesehen!

C.M. 
K. Vössing, Schreiben lernen, ohne lesen zu können? Zur Methode des antiken Elementarunterrichts, ZPE 123, 1998, 121-125.

Der durch eine Monographie einschlägig ausgewiesene Autor (Schule und Bildung im Nordafrika der römischen Kaiserzeit, CollLat 238, Brüssel 1997) wendet sich gegen die bei R. Cribiore (Writing, Teachers and Students in Graeco-Roman Egypt, American Studies in Papyrology 36, Atlanta/Georgia 1996, 129-152) geäußerte These, römische ABC-Schüler hätten häufig sofort nach dem Erlernen der Buchstaben Texte abschreiben müssen, ohne sie folglich auch lesen zu können. Vössing zeigt anhand von einschlägigen Texten, daß die paläographischen Beobachtungen, die Frau Cribiore zur Stützung ihrer Ansicht herbeiziehen zu können meinte, nicht durch textliche Belege ergänzt werden können (S. 123f.). Im vorliegenden Beitrag werden sie daher durch "ein Auseinanderklaffen von Theorie und Praxis" erklärt.

C.M. 